\title{
A Review of Wound Dressing Practices
}

\author{
Ilenghoven D1,2*, Chan C Y1, Wan Ahmad Kamal WSR ${ }^{1,2}$, \\ MohdYussof SJ1,2 and Ibrahim S1 \\ ${ }^{1}$ Department of Plastic and Reconstructive Surgery, Hospital Sungai Buloh, Malaysia \\ ${ }^{2}$ Discipline of Plastic and Reconstructive Surgery, Faculty of Medicine, UITM, Malaysia
}

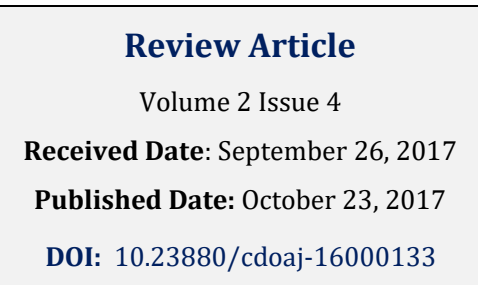

*Corresponding author: Devananthan Ilenghoven, University of Technology MARA, UITM Campus Sungai Buloh road hospital, 47000 Sungai Buloh, Malaysia, Tel: +60172296925; E-mail: i.devanathan@gmail.com

\section{Abstract}

For decades, the wound dressing was purely presented as-, and provided a physical barrier against external injuries and contaminants - a 'passive' yet elemental part in wound healing. Today, the suggestion that wound dressing works simply as a physical protection in the wound management sector is only seen as a precedent. Now, wound dressings when applied correctly take on the active role which supports wound closure. Having spent billions of dollars and endless hours poured into research geared toward the development of the latest wound dressing innovations; the current marketplace is more than capable to offer a broad spectrum of products formulated with almost any combination of properties and claimed benefits. As a result, this can be quite overwhelming for numerous wound care professionals. One way to get this situation streamlined is by simply categorising wound dressings according to their principal components: gauze, film, hydrogel, foam, hydrocolloid, or alginate dressings. Dressings within a given category may offer many similar features and can therefore be grouped accordingly. Conversely, since composite wound dressings are a combination of multiple layers contributing more than one function in wound care management, these should instead be categorised according to the clinically predominant component. Antimicrobial agents such as silver or iodine can be permeated into virtually all wound dressing categories. To comprehend the specific types of wound dressing, wound care professionals should be obligated to keep abreast with the latest technological advancement in wound dressing.

Keywords: Wound Dressing; Dressing Techniques; Materials; Wound care

Abbreviations: MVTR: Moisture Vapour Transmission Rate; PHMB: Polyhexamethylenebiguanide; VRE: Vancomycin-Resistant Enterococcus; MMPs: Matrix Metalloproteinases; EGF: Epidermal Growth Factor; NPWT: Negative Pressure Wound Therapy; VAC: Vacuum Assisted Closure.

\section{Introduction}

There are dressing techniques and materials aplenty for management of both acute and chronic non-healing wounds. Manufacturers have been developing modern dressings to manipulate the wound environment. That said, with a hugely diversified range of products available in the current market, the selection process for dressings for any wound can be quite a daunting task. In everyday practice, the standard choice of dressings is often based on familiarity, personal preference and cost.

Our civilization has been covering wounds with a variety of materials since the earliest written records. Sumerian cuneiform tablets before 2000 BCE describe the 


\section{Clinical Dermatology Open Access Journal}

application of poultices formed of mud, milk, and plants to wounds, and Egyptian papyruses from 1550 and 1650 BCE provide specific details of how to wash the wound, prepare and apply plasters of honey, plant fibers, and animal fat, and then bandage the wound [1]. Materials used to cover wounds since Egyptian times have only slowly evolved from readily available materials in nature to materials specifically designed by man to provide particular benefits for wound healing. In the past quarter century, the evolution of wound dressing materials has increased exponentially.

The Greek physician Galen (120-201A.D) had noted empirically that wounds heal optimally in a moist environment. In 1962, Winter noticed that occluded wounds required less time for epithelialization compared to wounds left open to air, which was supported by Cho and Lo, 1998 [2,3]. A closed dressing exposes the wound continuously to proteinases, chemotactic, complement \& growth factors in the surrounding fluid, which may be otherwise lost in case the wound is left exposed [4]. Even the electrical gradient required for stimulation of fibroblasts and epithelial cell migration is maintained and further trauma to the wound is avoided by this physical barrier.

In late 20th century, more clinical data was published in support of this and manufacturers began producing occlusive wound dressings, which were designed to preserve and protect a moist environment in the wound. Newer occlusive dressings speed up re-epithelialization, stimulate collagen synthesis, creates hypoxic environment at the wound bed to promote angiogenesis and decrease $\mathrm{pH}$ at wound surface, creating an environment inhospitable to bacterial growth, which decreases the rate of wound infection [5,6]. Moisture vapour transmission rate (MVTR) is an important consideration when evaluating dressings. The use of occlusive dressings, particularly on chronic wounds where bacterial overgrowth and therefore wound infection is a potential problem, initially raised concerns. Hutchinson, et al. reviewed the use of these dressings, comparing infection rates between occlusive and non-occlusive dressings in over 100 published studies finding that rates were $2.6 \%$ versus $7.1 \%(\mathrm{p}<0.001)$ in favour of occlusive dressings [7]. They have an edge over gauze dressings in terms of patient comfort, convenience and compliance as well as better cosmetic results because of reduced scarring [810].

The complexities of wound healing are still not fully understood. The wound dressing market has grown considerably over the last 25 years; however, few dressings have all the required characteristics for wound healing. The decision-making process to select the most appropriate dressing for the treatment of a wound can be complicated and clarity concerning dressing form and function is often a further challenge [11]. Prior to dressing selection, it is important to identify the purpose or principal aim of the proposed treatment. Dressing selection is only one part of a holistic wound management plan with individualised patient goals. It is necessary to assess the whole patient, diagnose underlying disease pathology and assess the patient's concerns before assessing the wound and choosing the dressing [12]. Effective wound management is not only about the availability and use of new dressings, it requires an understanding of the process of tissue repair and the knowledge of the properties of the dressings available.

\section{Wound Bed Preparation}

Wound bed preparation is also paramount to improve healing. An important factor in wound bed preparation is the maintenance of moisture balance, which often involves exudate management. Failure to manage exudate adequately can expose the peri-wound skin to toxic exudate that may impede healing [13]. The TIME principle was developed by an international advisory board on wound bed preparation [12]. The TIME principle is based on four factors: wound, clinical action, suggested product solution and the healing outcome (Table 1).

\begin{tabular}{|c|c|c|c|c|}
\hline $\begin{array}{c}\text { Clinical } \\
\text { observations }\end{array}$ & Proposed pathophysiology & $\begin{array}{c}\text { Wound bed preparation } \\
\text { clinical actions }\end{array}$ & $\begin{array}{c}\text { Effect of wound bed } \\
\text { preparation actions }\end{array}$ & Clinical outcome \\
\hline $\begin{array}{c}\text { Tissue non-viable on } \\
\text { deficient }\end{array}$ & $\begin{array}{c}\text { Defridement (episodic or } \\
\text { continuous): } \\
\text { debris impair healing }\end{array}$ & $\begin{array}{c}\text {-Autolytic, sharp surgical, } \\
\text { enzymatic, mechanical or } \\
\text { biological } \\
\text {-Biological agents }\end{array}$ & $\begin{array}{c}\text { Restoration of wound } \\
\text { base and functional } \\
\text { extracellular matrix } \\
\text { proteins }\end{array}$ & Viable wound base \\
\hline $\begin{array}{c}\text { Infection or } \\
\text { Inflammation }\end{array}$ & $\begin{array}{c}\text { High bacterial counts or } \\
\text { prolonged inflammation- }\end{array}$ & $\begin{array}{c}\text { Remove infected foci } \\
\text { Topical/systemic: }\end{array}$ & $\begin{array}{c}\text { Low bacterial counts or } \\
\text { controlled inflammation: }\end{array}$ & $\begin{array}{c}\text { Bacterial balance } \\
\text { and reduced }\end{array}$ \\
\hline
\end{tabular}




\begin{tabular}{|c|c|c|c|c|}
\hline & \begin{tabular}{|c|} 
increased Inflammatory \\
cytokines \\
-increased Protease activity \\
-decrease Growth factor \\
activity
\end{tabular} & $\begin{array}{l}\text {-Antimicrobials } \\
\text {-Anti-inflammatories } \\
\text {-Protease inhibition }\end{array}$ & $\begin{array}{l}\text {-decrease Inflammatory } \\
\text { cytokines } \\
\text {-decrease Protease } \\
\text { activity } \\
\text {-increase Growth factor } \\
\text { activity } \\
\end{array}$ & inflammation \\
\hline Moisture imbalance & $\begin{array}{l}\text { Desiccation slows epithelial } \\
\text { cell migration } \\
\text { Excessive fluid causes } \\
\text { maceration of wound margin }\end{array}$ & $\begin{array}{c}\text { Apply moisture-balancing } \\
\text { dressings } \\
\text { Compression, negative } \\
\text { pressure or other methods } \\
\text { of removing fluid }\end{array}$ & $\begin{array}{l}\text { Restored epithelial cell } \\
\text { migration, desiccation } \\
\text { avoided } \\
\text { Oedema, excessive fluid } \\
\text { controlled, maceration } \\
\text { avoided } \\
\end{array}$ & Moisture balance \\
\hline $\begin{array}{l}\text { Edge of wound - } \\
\text { non-advancing or } \\
\text { undermining }\end{array}$ & \begin{tabular}{|l} 
Non-migrating keratinocytes \\
Non-responsive wound cells \\
and abnormalities in \\
extracellular matrix or \\
abnormal protease activity
\end{tabular} & \begin{tabular}{|c|} 
Re-assess cause or consider \\
corrective therapies: \\
-Debridement \\
-Skin grafts \\
-Biological agents \\
-Adjunctive therapies \\
\end{tabular} & $\begin{array}{l}\text { Migrating keratinocytes } \\
\text { and responsive wound } \\
\text { cells. Restoration of } \\
\text { appropriate protease } \\
\text { profile }\end{array}$ & $\begin{array}{c}\text { Advancing edge of } \\
\text { wound }\end{array}$ \\
\hline
\end{tabular}

Table 1: TIME - Principles of wound bed preparation.

\section{Wound Dressings}

Dressings can be classified into passive, interactive, bioactive and tissue engineered dressings. Passive dressings are simple products like gauze which have no direct effect on the wound except protecting it. Active dressings actually promote healing through the creation of a moist wound environment. Most modern dressing products are interactive dressings as they interact with the wound bed to provide optimum environment at the wound dressing interface. The basic requirements of an appropriate wound dressing were explained by Scales in 1956 (Table 2).

\begin{tabular}{|c|}
\hline Characteristics of an ideal wound dressing \\
\hline Provide barrier to external contaminants \\
\hline Non adherent \\
\hline High capacity for absorption \\
\hline High moisture vapour permeability \\
\hline $\begin{array}{c}\text { Prevents capillary loops penetrating into dressing } \\
\text { material }\end{array}$ \\
\hline Capable of being sterilized \\
\hline Good adhesion to surrounding skin \\
\hline Hypoallergenic \\
\hline Comfortable to wear \\
Cost effective \\
\hline
\end{tabular}

Table 2: Characteristics of an ideal dressing.

\section{Inert/Passive Dressings}

For many years the dressings used were of the 'passive' concept including gauze, lint, non-stick and tulle. They fulfil very few of the properties of an ideal dressing and have very limited use as primary dressings, but some are useful as secondary dressings. Passive dressings are moisture- retaining thus speeds epithelisation of acute, superficial compared with air-exposed wounds [14]. Acute wound fluid under the occlusive bandage contains substances, such as growth factors, that stimulate proliferation of fibroblasts and endothelial cells, promoting granulation tissue formation [15-17]. In addition to gauze, lint and cotton dressings, other simple modified absorbent pads covered with a perforated plastic film to prevent adhering to a wound are used as primary and secondary dressings. They are used in minimal and low exudative wounds. Secondary dressing can be used over moderate to highly exudative wounds and over hydrocolloid paste, iodine, alginate and other primary dressings. Tulle/paraffin gauze dressings are among the earliest modern dressings. Many variations have been developed over the years by changing the loading of paraffin in the base. These dressings produce a waterproof paraffin cover over the wound, but this may lead to maceration as the water vapour and exudation may not pass through and be trapped within the wound. These dressings are permeable to bacteria, may adhere to the wound and in some cases may cause trauma on removal and will require a secondary dressing. Use is limited to simple clean superficial wounds and minor 
burns. They are also used as a primary dressing over skin grafts. There are modern alternative dressings composed of synthetic fibers tightly meshed and impregnated with materials that allow moisture to pass through and thus minimise any maceration of the wound and tissues [18].

\section{Interactive Dressings}

\section{Semi-Permeable Film Dressings}

Film dressings are adhesive, thin transparent polyurethane, which are permeable to gas but impermeable to liquid and bacteria. Films are elastic, conformable, less painful on removal and transparent allowing inspection of the wound [19]. As films are nonabsorbent they are not suitable for exudating wounds although island dressings with a central non-stick pad are available and can absorb slightly more exudate that the simple films [8]. Films can also be used as secondary dressings to waterproof a primary dressing such as foam. Film dressings generally require a border of dry, intact skin for the adhesive edge of the dressing; film dressings will not adhere to moist skin or moist wound beds because the moisture inactivates the adhesive. Therefore, the condition of the periwound skin should be assessed before application to determine if a film dressing is appropriate. Semi-permeable dressings trap moisture which creates a curative environment for granulating wounds besides allowing autolytic debridement of necrosis [20].

\section{Hypertonic Saline Dressings}

This group of dressing consist of cotton or gauze saturated with hypertonic sodium chloride (3-20\%) which are available in various forms. The hypertonic saline generates an osmotic action to cleanse the wound by wicking necrotic or infected purulent debris [21]. Bacterial growth is inhibited by hypertonic properties. As hypertonic saline dressings depend on wound moisture to moisten them, they are not appropriate for minimally draining wounds or wounds covered with dehydrated slough or eschar.

\section{Capillary Action/ Hydroconductive Dressings}

Capillary rapid action dressings are designed to wick exudate and microorganisms away from the wound surface. This group of dressing will effectively manage wound exudates and interstitial fluid levels and prevent maceration of the healthy tissue in and around the wound [22]. It will maintain a moist wound healing environment known to be beneficial to wound healing. The drawing in of exudates and interstitial fluid will promote autolytic debridement of necrotic and sloughy tissue. Excessive cytokines and proteases and reduced growth factors can be the cause of chronic wounds and may be attributable to wound inflammation and resultant fluids, therefore optimizing the fluid levels within a wound bed is crucial to successful healing $[23,24]$. It is a three-layer dressing designed to rapidly absorb exudates and interstitial fluids and optimize conditions for healing at the wounddressing interface. Rapid capillary action dressings have a central wicking layer that quickly distributes absorbed fluid throughout the dressing and create a sustained movement of fluid away from the wound bed. Fluid is drawn vertically into the dressing and there is no lateral movement of fluid at the wound interface which could cause maceration.

\section{Silicone Dressings}

Silicones are polymers with a structure that consists of alternate atoms of silicone and oxygen with organic groups attached to the silicone atoms. The degree of polymerisation determines the various physical forms of the silicone. Soft silicones are a particular form of solid silicones, which are soft and tacky. These properties enable the silicone to adhere to dry surfaces. A soft silicone dressing is coated with soft silicone as an adhesive or a wound contact layer. The intrinsic properties of soft silicone provide gentle adhesion and minimises wound and surrounding skin trauma at dressing change [25]. They have been shown to prevent trauma to the wound bed and periwound skin and have been described as 'atraumatic' for this reason. Soft silicone dressings have been available for over 10 years and were developed to minimise the problems of pain and trauma at dressing change, to protect the periwound skin and promote comfort during wear. They can be used on a wide range of low to highly exuding wounds and may be particularly suited to patients with fragile skin and/or those experiencing pain at wound dressing changes $[26,27]$. They have been shown to help prevent woundrelated complications and to minimise pain on removal in a range of wound types and patient groups. However, not all soft silicone dressings are the same and it is important that clinicians understand the difference between the products available in order to select the most appropriate dressing for the patient and the wound for optimal outcomes. Soft silicones also have a role in scar management and are used in the treatment of hypertrophic and keloid scars.

\section{Foam Dressings}

Foam dressings are highly absorbent polyurethane dressings, available as pads, sheets and cavity dressings. They create a moist environment and provide thermal insulation to the wound. They are non-adherent, easy to apply and remove and are meant for highly exuding 
wounds [28]. They can be layered in combination with other materials with overlying compression bandages. Foam dressings are also available with charcoal impregnation for malodorous wounds. Depending on the level of exudate, foams can be left in place for up to seven days. Foam wound cavity dressings reduce dead space in the wound, conform to wound shape and absorb large amounts of exudate, therefore reducing the need for frequent dressing changes although cavity foam dressings require secondary dressings and that adds to cost. Foams are generally non adhesive and require a secondary dressing or tape/bandage to keep in place. Foam dressings may be manufactured with an adhesive border, which eliminates the need for a securing device or without an adhesive border. Shaped versions of these dressings are also available. Foam dressings are also available which release agents such as antimicrobials, moisturizers or anti-inflammatory analgesics into the wound.

\section{Bio-active Dressings}

\section{Antimicrobial Dressings}

This group of dressing contains low concentrated, sustained release of antimicrobial agents to the wound bed aimed at providing optimum condition to support wound healing. This slower release with lower intensification reduces the risk for toxicity to host cells while reducing the wound bed bio-burden. Many factors must be taken into consideration while selecting the appropriate antimicrobial agent including capability to reduce or eradicate common micro-organisms, hypoallergen in nature, non-toxic to human cells, known sensitivity and reduced risk of developing resistant. This dressing helps with wounds with high risk of infections such as wounds communicating with internal organs, cavity or depth involving dermis and beyond.

The antimicrobial activity are provided by iodine, polyhexamethylene biguanide (PHMB) or silver incorporation. Different composition of products handles exudate distinctively. Antimicrobial dressing are widely available now impregnated in a variety of size, shape and delivery system.

\section{- Silver Impregnated Dressings}

Silver is well known as an antiseptic agent (silver nitrate and silver sulphadiazine) for ages. However, the delivery system in the form of a salt has been a limiting factor for its successful and widespread biological use. Recently, silver has been incorporated into different wound dressing products like gauzes, hydrocolloids, alginates, foams, creams and gels but each of them differ in the way in which silver ions are released. Silver nitrate and silver sulphadiazine release silver at concentration of 3200 ppm. Most pathogenic organisms are killed in-vitro at concentration of $10-40 \mathrm{ppm}$. The development of nanochemistry has produced micro fine particles which increase silver's solubility and releases silver ions in concentration of 70-100 ppm. Nanocrystalline silver system kills all microbes found in the wound including fungi, MRSA and vancomycin-resistant enterococcus (VRE) [29]. Silver absorbed by epidermal cells induces production of metallotheine which in turn increases uptake of zinc and copper, which increases RNA and DNA synthesis [30]. This then promotes cell proliferation and tissue repair [31]. In any case of non-healing chronic wound there is an excess of matrix metalloproteinases (MMP), which increases the inflammation and inflammatory cell exudates and degrade the growth factors. Nanocrystalline silver decreases the MMP activity both in-vivo and in-vitro because of its inhibitory effect on zinc activity, which is required for MMPs, as also its inhibitory effect on release of pro-inflammatory cytokines and tumour necrosis factor- alpha. The Acticoat dressing is a three layered dressing consisting of an absorbent rayon/polyester core laminated between upper and lower layer of silvercoated high density polyethylene mesh. The silver concentration on the wound surface is 20-30 times greater than the concentration required to kill microbes. These nanocrystalline dressings can be left in place for up to 5-7 days, which avoids trauma to the new epithelial growth.

\section{- Cadexomer Iodine Dressings}

Iodine has been speculated delay healing and is cytotoxic, however, there is substantial evidence to suggest that the commonly-used low concentration, slow release iodophors boosts the rate of healing and are effective as highly potent antimicrobials on a larger scale of activity; and that includes MRSA, an antibiotic-resistant strain. The reputation of iodine is now widely accepted that slowreleasing iodophor antimicrobials are safe and have minimal detrimental impact on wound healing [32]. When applied to the wound, cadexomer iodine based products absorb fluids, removing exudate, pus and debris. As they swell, iodine is slowly released killing micro-organisms and forming a protective gel over the wound surface. Sustained release iodine from cadexomer iodine dressing does not cause cytotoxic effects which are seen with povidone iodine. Cadexomer iodine contains $0.9 \%$ iodine in prepation and delivered at $0.1 \%$ concentration to tissue which are rarely toxic. This dressing is prepared as a spherical beads with three dimensional polysaccharide lattice. Another common iodine preparation is Iodosorb, an autolytic debriding agent with broad spectrum antimicrobial. They are best used for heavy exudative 
wounds due to its absorptive capability above six times its weight. It is contraindicated in patients with sensitivity to iodine and not recommended for pregnant or lactating women. There has been anecdotal evidence that some patients have experienced osmotic pain. Cadexomer iodine dressings are commonly used in the treatment of chronic, non-healing wounds including leg ulcers (venous, arterial and mixed aetiology), pressure ulcers and exuding, infected wounds (in conjunction with systemic antibiotics).

\section{Hydrogel Dressings}

Hydrogel dressings are water- or glycerin-based products. Because they are usually clear or transparent, the wound can be monitored without removing the dressing. Use hydrogels to maintain a moist wound environment on a clean, healthy, granulating wound and to facilitate autolytic debridement in wounds with necrotic tissue such as slough or eschar. Hydrogels can be used on pressure ulcers, skin tears, surgical wounds, and burns, including radiation oncology burns and are safe on neonatal skin. These dressings are suitable for wounds with minimal to moderate exudate. Hydrogel dressings are commonly available in three forms: amorphous gel, impregnated-gauze, and sheet hydrogel and can be useful when managing painful wounds. They rehydrate the wound bed, reduce pain because of their cooling effect, are non-adhesive, fill dead spaces, are easy to apply and remove [33-35]. They are best suited for dry wounds or those with minimal exudates. But they require a secondary dressing. Hydrogel dressings that act as a release platform have also been developed containing hyaluronic acid, antimicrobials and antibiotics. Oxyzyme for example is a two component hydrogel dressing which releases both oxygen and iodine into the wound and is suitable for non-infected moderately exudating wounds. Hydrogels can remain in-situ for up to three days. For easy removal of hydrogels, the wound is irrigated.

\section{Hydrocolloid Dressings}

Hydrocolloid dressings consist of absorptive ingredients (typically carboxymethylcellulose, pectin or gelatine). Like hydrogels, hydrocolloids can absorb minimal to moderate amounts of drainage and are suitable for partial- or full-thickness acute and chronic wounds. As they are occlusive, hydrocolloid dressings do not allow water, oxygen, or bacteria into the wound. This may help facilitate angiogenesis and granulation [36]. A hydrocolloid dressing creates an acidic environment which inhibits bacteria growth, promotes autolytic debridement in wounds with necrotic or sloughy tissue and helps with granulation or epithelialisation. However, because of their occlusive nature, hydrocolloids should not be used if the wound or surrounding skin is infected and are not recommended for use in diabetic foot ulceration. Hydrocolloid dressings are conformable to the patient's body and adhere well to high friction areas, such as the sacrum and heels. In some cases, hydrocolloid dressings may produce a distinctive odour, this is usually due to product breakdown and not infection. Hydrocolloid dressings carries risk of hypergranulation when used for exudative wounds for long periods.

\section{Hydrofiber Dressings}

This is a variant on hydrocolloid with extra absorbent properties, absorbing up to 25 times its own weight in fluid before losing its integrity. The dressings contain sodium carboxymethyl cellulose. They conform to the wound surface, are highly absorbent and interact with wound exudates to form a gel. They thus maintain a moist environment and allow autolytic debridement. They are indicated for pressure ulcers, lower limb ulcers and surgical wounds. The vertical wicking of exudate reduces maceration of surrounding skin. The dressings are claimed to be more absorbent than alginates and to promote non-traumatic dressing removal.

\section{Alginate Dressings}

Alginates (calcium or calcium/sodium) are highly absorbent, biodegradable dressings derived from seaweed. When placed over a moist wound, an ion exchange reaction occurs between calcium in the alginate and sodium in the wound fluid producing soluble calcium -sodium alginate -- a gelatinous mass, which helps in maintaining moist environment and facilitates autolytic debridement [37]. They conform to the shape of the wound and should be cut according to the shape of wound because if larger they can cause periwound maceration because of their tendency to absorb fluid across entire surface (lateral wicking) [38]. They are used as fillers for undermined and tunnelled wounds. They may leave fibrous debris in the wound, which is claimed to get biodegraded yet there have been reports of them causing long-term foreign body type reactions [39]. Calcium dressings need moisture/exudate from the wound to function; therefore they are not suitable for dry wounds or wounds with hardened eschar. The fibrous nature of most alginates can leave residual fibres in the wound if there is insufficient wound exudate to gel the fibres. This may precipitate an inflammatory reaction as it stimulates a foreign body response. Caution is also needed when using alginate rope dressings in very deep or narrow sinuses, as complete removal can be difficult. These dressings are capable of absorbing up to 20 times their weight in fluid, and can be used in infected and uninfected wounds. Alginates require a secondary dressing; foams or 
hydrocolloids will secure the alginate and keep it from drying out. If the wound is infected, the secondary dressing should be non-occlusive. The calcium component of the dressing acts as a hemostat and can be useful in managing wound bleeding.

\section{Honey Dressing}

Honey has been used as a wound dressing for thousands of years, but only in more recent times has a scientific explanation become available for its effectiveness. It is now realized that honey is a biologic wound dressing with multiple bioactivities that work in concert to expedite the healing process. The physical properties of honey also expedite the healing process: its acidity increases the release of oxygen from hemoglobin thereby making the wound environment less favorable for the activity of destructive proteases, and the high osmolarity of honey draws fluid out of the wound bed to create an outflow of lymph as occurs with negative pressure wound therapy $[40,41]$. Honey has a broadspectrum antibacterial activity, but there is much variation in potency between different honeys. There are 2 types of antibacterial activity. In most honeys the activity is due to hydrogen peroxide, but much of this is inactivated by the enzyme catalase that is present in blood, serum, and wound tissues [42]. Inmanuka honey, the activity is due to methylglyoxal which is not inactivated. The manuka honey used in wound-care products can withstand dilution with substantial amounts of wound exudate and still maintain enough activity to inhibit the growth of bacteria [43]. There is good evidence for honey also having bioactivities that stimulate the immune response (thus promoting the growth of tissues for wound repair), suppress inflammation, and bring about rapid autolytic debridement.

\section{Chitosan Dressing}

Chitosan is a natural biopolymer that is derived from chitin, a major component of crustacean outer skeletons. This material is known in the wound management field for its haemostatic properties. Further, it also possesses other biological activities and affect macrophage function that helps in faster wound healing [44]. It also has an aptitude to stimulate cell proliferation and histoarchitectural tissue organization [45]. The biological properties including bacteriostatic and fungistatic properties are particularly useful for wound treatment. Flexible, thin, transparent, novel chitosan-alginate polyelectrolyte complex membranes caused an accelerated healing of incision. Healing at split skin graft donor sites was studied with chitosan by dressing half with chitosan and half with a conventional dressing. It showed that chitosan facilitated rapid wound re- epithelialization and the regeneration of nerves within a vascular dermis. Early returns to normal skin color at chitosan-treated areas were demonstrated [46]. Treatment with chitin and chitosan demonstrated a substantial decrease in treatment time with minimum scar formation on various animals [47]. Chitosan provides a non-protein matrix for 3D tissue growth and activates macrophages for tumoricidal activity. It stimulates cell proliferation and histoarchitectural tissue organization. Chitosan is a hemostat, which helps in natural blood clotting and blocks nerve endings reducing pain. Chitosan will gradually depolymerize to release N-acetyl-b-Dglucosamine, which initiates fibroblast proliferation and helps in ordered collagen deposition and stimulates increased level of natural hyaluronic acid synthesis at the wound site. It helps in faster wound healing and scar prevention.

\section{Collagen Dressing}

Collagen, the protein that gives the skin its tensile strength, plays a key role in each phase of wound healing. It attracts cells, such as fibroblasts and keratinocytes, to the wound, which encourages debridement, angiogenesis, and re-epithelialization [48]. In addition, collagen provides a natural scaffold or substrate for new tissue growth. Collagen dressings stimulate new tissue growth and encourage the deposition and organization of newly formed collagen fibers and granulation tissue in the wound bed [49]. These dressings chemically bind to matrix metalloproteinases (MMPs) found in the extracellular fluid of wounds. MMPs normally attack and break down collagen, so it's thought that wound dressings containing collagen give MMPs an alternative collagen source, leaving the body's natural collagen available for normal wound healing. There are a number of different collagen dressings available, which employ a variety of combining agents such as gels, pastes, polymers, oxidized regenerated cellulose, and ethylene diamine tetra acetic acid. The collagen within these products tends to be derived from bovine, porcine, equine, or avian sources, which is purified in order to render it non antigenic. The collagen in a given collagen dressing can vary in concentration and type. Certain collagen dressings are comprised of Type I (native) collagen; whereas, other collagen dressings contain denatured collagen as well. A given collagen dressing may contain ingredients, such as alginates and cellulose derivatives that can enhance absorbency, flexibility, and comfort, and help maintain a moist wound environment. Collagen dressings have a variety of pore sizes and surface areas, as well. All of these attributes are meant to enhance the wound management aspects of the dressings. Many collagen dressings contain an antimicrobial agent to control pathogens within the 
wound. Collagen dressings typically require a secondary dressing. They have shown excellent results in helping to heal partial thickness wounds, necrotic wounds, skin grafts and second-degree burns. Depending on the type of wound they are being used on, collagen dressings should be changed about every seven days to remain effective.

\section{Silk Based Wound Dressings}

Silkworm silk fibers are composed of two fibrous protein microfilaments (fibroins) embedded in a glue-like glycoprotein coating (sericin) and can be isolated from the silkworm Bombyxmori and Antheraeamylitta. The sericins ensure the cohesion of the cocoon by sticking the twin filaments together and constitute $25-30 \%$ of the weight of the fiber. Finally the fiber is coated with a variety of other proteins that protects the cocoon. It is getting more and more attention due to its good biocompatibility, slow degradability, hemocompatibility, and high mechanical strength, although silk sericin might be immunogenic for patients [50]. This new wound dressing, a wax-coated silk fibroin woven fabric functioned as a non adhesive layer, while the sponge made of sericin and glutaraldehyde-cross-linked silk fibroin/gelatin acts as a bioactive layer. The wax-coated silk fibroin fabrics showed improved mechanical properties less adhesive characteristics than the commercial wound dressing [51]. The sericin-silk fibroin/gelatin spongy bioactive layers showed homogeneous porous structure and controllable biodegradation depending on the degree of cross-linking. Furthermore, they proved that fibroin can improve wound healing by supporting of attachment of epidermal cells and fibroblasts spreading and proliferation [52]. The wounds treated with the bilayered wound dressings showed more reduction of wound area and improved epithelialization as well as collagen formation in fullthickness wounds. There exist transgenic silkworms (Bombyxmori) that can combine their silk thread with human growth factors and supports angiogenesis. It has been shown that there was no cytotoxic effect of the silks and growth factors improved the proliferation of mesenchymal stem cells. Silk mats made of porous, structured electrospunnano scaled silk fibers containing epidermal growth factor (EGF) has high affinity receptors expressed in both fibroblasts and keratinocytes and has been shown to accelerate wound healing in vivo by stimulation of proliferation and migration of keratinocytes. Silk biomaterial wound dressings increases wound healing rate, including re-epithelialization, dermis proliferation, collagen synthesis, and reduced scar formation.

\section{Deodorizing Dressings}

Wound odour can be a significant problem for patients, particularly those with non-healing or malignant wounds. Charcoal based products can be effective in reducing wound odour due to its ability to absorb gas molecule. Activated charcoal is used to absorb odour and is designed to be used as a secondary dressing or within a bandage system. A range of other composite dressings containing activated charcoal exists, some containing silver, which can be used as an absorbent wound contact layer managing both odour and exudate. As odour is usually due to bacteria, particularly anaerobic, Metronidazole Gel can also be effective as a topical odour reducing agent $[53,54]$.

\section{Tissue Engineered Skin Substitutes}

\section{Biological or Synthetic Matrix Dressings}

Skin substitutes have been used as dressings which protect wound from fluid loss and infection and others are being used as wound implants, which help replace extracellular matrix molecules or deliver various growth factors to prepare bed for permanent skin coverage. These artificial engineered scaffolds for wound healing are mimicking structure and functions to native extracellular matrix. They can be created by bacteria producing cellulose, by cultured transgenic silkworms or by several electrospinning techniques. The last one is an efficient technique to produce a variety of polymeric nanofibers. Electrospun scaffolds for wound healing provide high surface area to volume ratio that promotes cell-matrix interaction at the nanoscale [55,56]. Electrospun collagen is the most biomimetic nanofibrous scaffold due to its structure and origin closely similar to the native extracellular matrix of the skin, but also its alternative, gelatine, and other natural polymers including silk fibroin, chitin/chitosan, fibrinogen, and different blends have been electrospun as wound dressing or cellular matrix and have been demonstrated to be efficient to cell attachment, growth, and cell infiltration in in vitro culture [57]. Artificial created scaffolds also can be combined and added with different components like antibiotics or antibacterial drugs, anaesthetics like lidocaine, or silver particles that are spun together with the synthetic nanofibers [58,59]. These combined scaffolds work as drug delivery systems and are releasing a controlled rate of the drugs and therefore might have great potential in different applications in wound healing.

Electrospunnanofibrous polyurethane membrane: Electrospinning is a process by which nanofibers can be produced by an electrostatically driven jet of polymer solution. Electrospun fibers are collected in the form of membranes. The porous structured electrospun 
membrane is particularly important for its favorable properties: it exudates fluid from the wound, does not build up under the covering, and does not cause wound desiccation. The electrospunnanofibrous membrane shows controlled evaporative water loss, excellent oxygen permeability, and promote fluid drainage ability, but still it can inhibit exogenous microorganism invasion because its pores are ultra-fine. Histological examination indicates that the rate of epithelialization is increased and the dermis becomes well organized if wounds are covered with electrospunnanofibrous membrane.

\section{Extracellular matrix-based wound dressing:}

Extracellular matrix is critical to wound healing, as it serves not only as a scaffold, supporting the migration of cells and the ingrowth of blood vessels, but also as an activator, coordinator, and director of the healing process. For example, Oasis, a matrix produced from pig small intestine submucosa, is derived from extracellular matrixbased wound product that is compatible with human tissue. Components of the extracellular matrix that are retained in Oasis include glycosaminoglycans, proteoglycans, fibronectin and other matrix-associated factors such as fibroblast growth factors. It is a thin, transparent layer that is recommended for use in all partial and full thickness wounds and skin loss injuries such as superficial and full thickness burns.

Collagen and oxidized regenerated cellulose matrix: Promogran, a sterile, freeze dried matrix composed of collagen and oxidised regenerated cellulose, formed into a sheet approximately $3 \mathrm{~mm}$ thick cut into hexagonal pieces. The matrix absorbs liquid and forms a soft, conformable, biodegradable gel that physically binds and inactivates matrix metalloproteases. It is recommended for all types of chronic wounds that are free of necrotic tissue and show no signs of infection. Once in place it must be covered with a low-adherent secondary dressing to maintain a moist wound healing environment. It can be used in conjunction with standard compression therapy when treating venous ulcers. Frequency of dressing change will depend on the level of exudate. The matrix absorbs exudate and forms a soft biodegradable gel, which rebalances the wound environment. The gel binds and inactivates matrix metalloproteinases, which when present in excessive levels, have a detrimental effect on wound healing as they damage regenerating tissue.

Integra dermal regeneration template: Integra dermal regeneration template is comprised of a porous collagen/chondroitin- 6 sulfate matrix overlaid with a thin silastic sheet, which acts as a scaffold for dermal regeneration. Its unique action inhibits granulation and promotes the growth of neo-dermis through the collagen and glycosaminoglycan matrix. The silastic layer provides a temporary epithelial covering, which is removed prior to secondary grafting with a thin split-thickness autograft or cultured keratinocyte sheet. When placed on a surgically debrided or excised wound, it provides the needed framework for the blood vessels and dermal skin cells to remodel the damaged site. As skin cells migrate into the matrix, the collagen is slowly absorbed and replaced with collagen produced from the person's own cells. In approximately 14 to 21 days, the scaffold is eventually remodeled as the patient's cells rebuild the damaged site. Complete wound closure occurs as epidermal cells migrate from the wound edges. For larger wounds, a thin skin graft of the person's epidermis may be applied to the wound area to facilitate complete wound closure. Wound closure is typically complete within 30 days. A person is left with a healed wound created from their own tissue.

\section{Negative Pressure Wound Therapy}

Negative pressure wound therapy (NPWT) or vacuum assisted closure (VAC) is a significant, clinically proven advancement in wound care that promotes active wound healing at the cellular level through negative pressure. Negative pressure applies non-compressive mechanical forces to the tissue, which allows arterioles to dilate and increases blood flow. NPWT produces mechanical stress and creates a pressure gradient across tissues. In addition to increasing blood flow, this places physical strain on cells that stimulates interaction with soluble growth factors in the extracellular matrix. The effect is often referred to as cell stretch. Cell stretch encourages cell proliferation and maturation, resulting in improved vessel growth, collagen deposition, and granulation tissue formation. Removal of interstitial fluid reduces edema and decompresses tissue, allowing for improved capillary perfusion and angiogenesis. This action increases the oxygen and nutrient supply, as well as access for white blood cells important to the healing process. Excess exudate contains microorganisms, debris, proteolytic enzymes and other components that prolong inflammation, cause edema and delay healing. Removing this excess exudate through vacuum assisted drainage aids in microbial control, while maintaining a moist environment for important for healing. In its simplest form localized negative pressure is applied to a closed foam or gauze-based wound dressing fitted to a cavity wound sealed under a film dressing. A number of commercial systems are available. Depending on the system chosen and the type of wound filler, a negative pressure of between $80 \mathrm{mmHg}$ and $150 \mathrm{mmHg}$ is 
maintained by a portable pump, exudate being collected from the wound in a sealed reservoir. Recent developments include the inclusion of a closed wound irrigation mechanism within the system, which allows the wound to be intermittently flushed with an antimicrobial solution. Contraindications to this therapy exist in wounds that have: fistulae to organs or cavities; malignancy; untreated osteomyelitis; necrotic tissue in eschar; exposed blood vessels, nerves and anastomotic sites. In these situations, such therapy can lead to anastomotic breakdown, bleeding, pain and deterioration in the underlying untreated pathology.

\section{Conclusion}

An understanding of the basic principles and a systematic assessment of wound is important as all wound heals differently. Wound management is more than the application of a dressing and for many this remains a challenge simply because the choice of dressings is so vast. Dressing selection depends on the practitioner's skilled assessment of the wound and his knowledge of how to provide this optimum wound healing environment through the use of modern interactive dressings. For new advanced dressings 'bioactivity' appears to be the way forward in maintaining a moist healing environment, offering antimicrobial properties and cellular interactions. Wound dressing will continue to advance rapidly thus clinicians should try to obtain the maximum benefits from this evolving technologies and therapies.

\section{References}

1. Majno G (1975) The healing hand: man and wound in the ancient world. Harvard University Press.

2. Winter GD (1962) Formation of scab and rate of epithelialization of superficial wounds in the skin of the young domestic pig. Nature 193: 293-294.

3. Cho CY, Lo JS (1998) Dressing the part. Dermatol Clin 16(1): 25-47.

4. Moon CH, Crabtree TG (2003) New wound dressing techniques to accelerate healing. Curr Treat Options Infect Dis 5: 251-260.

5. V arghese MC, Balin AK, Carter DM, Caldwell D (1986) Local environment of chronic wounds under synthetic dressings. Arch Dermatol 122(1): 52-57.

Ilenghoven D, et al. A Review of Wound Dressing Practices. Clin Dermatol ] 2017, 2(4): 000133.
6. Alvarez OM, Mertz PM, Eaglstein WH (1983) The effect of occlusive dressings on collagen synthesis and re-epithelialization in superficial wounds. J Surg Res 35(2): 142-148.

7. Hutchinson JJ, McGuckin M (1990) Occlusive dressings: a microbiologic and clinical review. Am J Infect Control 18(4): 257-268.

8. Rubio PA (1991) Use of semiocclusive, transparent film dressings for surgical wound protection: An experience in 3637 cases. Int Surg 76(4): 253-254.

9. Jones AM, San Miguel L (2006) Are modern wound dressings a clinical and cost effective alternative to the use of gauze? J Wound Care 15(2): 65-69.

10. Robson MC, Smith PD (2001) Topical use of growth factors to enhance healing. In: Falanga $V$ (Eds.), Cutaneous wound healing. London: Martin Dunitz, Ltd, pp: 379-398.

11. Carville K (1999) General principles of wound management. In: The care of wounds: a guide for nurses. Oxford: Blackwell Science, pp: 49-67.

12. Schultz GS, Sibbald RG, Falanga V, Stacey M, Ayello EA, et al. (2003) Wound bed preparation: a systematic approach to wound management. Wound Repair Regen 11(1): S1-S28.

13. Ovington LG, Pierce B (2001) Wound dressings: form, function, feasibility, and facts. In: Krasner D, Rodeheaver G, et al. (Eds.). Chronic wound care: a clinical sourcebook for healthcare professionals. Wayne: Health Management Publications Inc, pp: 311-319.

14. Hinman CD, Miabach H (1963) Effect of air exposure and occlusion on experimental human skin wounds. Nature 200: 377-378.

15. Katz MH, Alvarez AF, Kirsner RS, Eaglstein WH, Falanga V (1991) Human wound fluid from acute wounds stimulates fibroblast and endothelial cell growth. J Am Acad Dermatol 25(6 Pt 1): 1054-1058.

16. Chen WY, Rogers AA, Lydon MJ (1992) Characterization of biologic properties of wound fluid collected during early stages of wound healing. J Invest Dermatol 99(5): 559-564.

17. Alper JC, Tibbetts LL, Sarazen AA Jr (1985) The in vitro response of fibroblasts to the fluid that 


\section{Clinical Dermatology Open Access Journal}

accumulates under a vaporpermeable membrane. J Invest Dermatol 84: 513-515.

18. Fan K, Tang J, Escandon J, Kirsner RS (2011) State of the art in topical wound- healing products. Plast Reconstr Surg 127(Suppl 1): 44S-59S.

19. Persson K, Salemark L (2000) How to dress donor sites of split thickness skin grafts: a prospective randomized study of four dressings. Scand J Plast Reconstr Surg Hand Surg 34(1): 55-59.

20. Murphy PS, Evans GR (2012) Advances in Wound Healing: A Review of Current Wound Healing Products. Plast Surg Int 2012: 190436.

21. Ji H, Wang L, Zhang Z, Jin B, Lin J, et al. (2009) Hypertonic saline soaks in treatment of burned skin. Burns 35(1): 152-153.

22. Ochs D, Uberti MG, Donate GA, Abercrombie $M$, Mannari RJ, et al. (2012) Evaluation of mechanisms of action of a hydroconductive wound dressing, Drawtex, in chronic wounds. Wounds 24: 9-3.

23. Livingston M, Wolvos $T$ (2011) Hydroconductive debridement: a new perspective in reducing slough and necrotic tissue. Presented at the $24^{\text {th }}$ Annual Symposium on Advanced Wound Care and the Wound Healing Society Meeting, Dallas, TX.

24. Wolcott RD, Cox S (2012) The effects of a hydroconductive dressing on wound biofilm. Presented at a Symposium of Investigators, Innovations for Wound Bed Preparation: The Role of Drawtex Hydroconductive Dressings, Tampa, FL.

25. Dykes PJ, Heggie R (2003) The link between peel force of adhesive dressings and subjective discomfort in volunteer subjects. J Wound Care 12(7): 260-262.

26. Gotschall CS, Morrison MI, Eichelberger MR (1998) Prospective, randomized study of the efficacy of 'Mepitel' on children with partial-thickness scalds. J Burn Care Rehabil 19(4): 279-283.

27. Platt AJ, Phipps A, Judkins K (1996) A comparative study of silicone net dressing and paraffin gauze dressing in skin-grafted sites. Burns 22(7): 543-545.

28. Anderson KE, Franken CPM, Gad P, Larsen AM, Larsen JR, et al. (2002) A randomized, controlledstudy to compare the effectiveness of two foam dressings in the management of lower leg ulcers. Ostomy Wound Manage 48(8): 34-41.

29. Lansdown AB (2006) Silver in health care: Antimicrobial effects and safety in use. Curr Probl Dermatol 33: 17-34.

30. Lansdown AB (2002) Metallothioneins: Potential therapeutic aids for wound healing in the skin. Wound Repair Regen 10(3): 130-132.

31. Demling RH, Leslie De Santi (2002) The rate of reepithelialization across meshed skin grafts is increased with exposure to silver. Burns 28(3): 264266.

32. Sibbald RG, Leaoer DJ, Queen D (2011) Iodine Made Easy. Wounds International 2(2).

33. Jones V, Milton T (2000) When, how to use Hydro gels. Nurs Times 96(23 Suppl): 3-4.

34. Lay-Flurrie K (2004) The properties of Hydrogel dressings and their impact on wound healing. Prof Nurse 19(5): 269-273.

35. Falanga V (2002) Wound bed preparation and the role of enzymes: A case for multiple actions of therapeutic agents. Wounds 14(2): 47-74.

36. Chvapil M, Holubec H, Chvapil T (1991) Inert wound dressings is not desirable. J Surg Res 51(3): 245-252.

37. Kannon GA, Garrett AB (1995) Moist wound healing with occlusive dressings: A clinical review. Dermatol Surg 21(7): 583-590.

38. Argar MS (1996) Four alginate dressings in the treatment of partial thickness wounds: A comparative experimental study. Br J PlastSurg 49(2): 129-134.

39. Suzuki Y, Tanihara M, Nishimura Y, Suzuki K, Yamawaki Y, et al. (1999) In vivo evaluation of a novel alginate dressing. J Biomed Mater Res 48(4): 522-527.

40. Kaufman T, Eichenlaub EH, Angel MF, Levin M, Futrell JW (1985) Topical acidification promotes healing of experimental deep partial thickness skin burns: a randomised double-blind preliminary study. Burns Incl Therm Inj 12(2): 84-90.

41. Greener B, Hughes AA, Bannister NP, Douglass J (2005) Proteases and $\mathrm{pH}$ in chronic wounds. J Wound Care 14(2): 59-61. 
42. Molan PC (1992) The antibacterial activity of honey: 1. The nature of the antibacterial activity. Bee World 73(1): 5-28.

43. Adams CJ, Manley-Harris M, Molan PC (2009) The origin of methylglyoxal in New Zealand manuka (Leptospermum scoparium) honey. Carbohydr Res 344(8): 1050-1053.

44. Balassa LL, Prudden JF (1984) Applications of chitin and chitosan in wound healing acceleration, in Chitin, Chitosan and Related Enzymes, Academic Press, San Diego, pp. 296-305.

45. Muzzarelli RAA (1989) Amphoteric derivatives of chitosan and their biological significance, in Chitin and Chitosan, Elsevier Applied Science, London, p. 8799.

46. Minami S, Okamoto $\mathrm{Y}$, Hamada $\mathrm{K}$, Fukumoto $\mathrm{Y}$, Shigemasa Y (1999) Veterinary practice with chitin and chitosan. EXS 87: 265-277.

47. Muzzarelli RA1, Mattioli-Belmonte M, Pugnaloni A, Biagini G (1999) Biochemistry, histology and clinical uses of chitins and chitosans in wound healing. EXS 87: 251-264.

48. Purna SK, Babu M (2000) Collagen based dressings: A review. Burns 26(1): 54-62.

49. Ovington L (2005) The art and science of wound dressings in the twenty-first century. In: Falabella AF, Kirsner RS (Eds.), Wound Healing. Boca Raton, Fla: Taylor \& Francis Group, p. 1-7.

50. Karahaliloğlu Z, Ercan B, Denkbaș EB, Webster TJ (2015) Webster, Journal of Biomedical Materials Research Part A 103(1): 135-144.

51. Chen FJ, Porter D, Vollrath F (2012) Morphology and structure of silkworm cocoons. Materials Science \&
Engineering C-Materials for Biological Applications 32: $772-778$.

52. Yu K, Lu F, Li Q, Zou Y, Xiao Y, et al. (2017) Accelerated wound-healing capabilities of a dressing fabricated from silkworm cocoon. Int J Biol Macromol 102: 901-913.

53. Newman V, Allwood M, Oakes RA (1989) The use of metronidazole gel to control the smell of malorous lesions. Palliat Med 3(4): 303.

54. Chiwenga S, Dowlen H, Mannion S (2009) Audit of the use of sugar dressings for the control of wound odor at Lilongwe Central Hospital, Malawi. Trop Doct 39(1): 20-22.

55. Tan W, Krishnaraj R, Desai TA (2001) Evaluation of nanostructured composite collagen-Chitosan matrices for tissue engineering. Tissue Eng 7(2): 203210.

56. Zhang R, Ma PX (2000) Syntheticnano-fibrillar extracellular matrices with predesigned macroporous architectures. J Biomed Mater Res 52(2): 430-438.

57. Zhong SP, Zhang YZ, Lim CT (2010) Tissue scaffolds for skin wound healing and dermal reconstruction. Wiley Interdiscip Rev Nanomed Nanobiotechnol 2(5): 510-525.

58. Trovatti E, Silva NH, Duarte IF, Rosado CF, Almeida IF, et al. (2011) Biocellulose membranes as supports for dermal release of lidocaine. Biomacromolecules 12(11): 4162-4168.

59. Nguyen TH, Kim YH, Song HY, Lee BT (2011) Nano Ag loaded PVA nano-fibrous mats for skin applications. J Biomed Mater Res B Appl Biomater 96(2): 225-233. 